\title{
Bezafibrate lowers very long-chain fatty acids in X-linked adrenoleukodystrophy fibroblasts by inhibiting fatty acid elongation
}

\author{
Marc Engelen • Martin J. A. Schackmann • \\ Rob Ofman • Robert-Jan Sanders • \\ Inge M. E. Dijkstra $\cdot$ Sander M. Houten • \\ Stéphane Fourcade • Aurora Pujol • \\ Bwee Tien Poll-The • Ronald J. A. Wanders • \\ Stephan Kemp
}

Received: 16 January 2012 / Revised: 14 February 2012 / Accepted: 22 February 2012

(C) The Author(s) 2012. This article is published with open access at Springerlink.com

\begin{abstract}
X-linked adrenoleukodystrophy (X-ALD) is caused by mutations in the $A B C D 1$ gene encoding ALDP, an ATPbinding-cassette $(\mathrm{ABC})$ transporter located in the peroxisomal membrane. ALDP deficiency results in impaired peroxisomal $\beta$-oxidation and the subsequent accumulation of very longchain fatty acids (VLCFA; > C22:0) in plasma and tissues. VLCFA are primarily derived from endogenous synthesis by ELOVL1. Therefore inhibiting this enzyme might constitute a feasible therapeutic approach. In this paper we demonstrate that bezafibrate, a PPAR pan agonist used for the treatment of
\end{abstract}

Communicated by: K. Michael Gibson

Electronic supplementary material The online version of this article (doi:10.1007/s10545-012-9471-4) contains supplementary material, which is available to authorized users.

M. Engelen · M. J. A. Schackmann · R. Ofman · R.-J. Sanders •

I. M. E. Dijkstra $\cdot$ S. M. Houten $•$ R. J. A. Wanders $\cdot$ S. Kemp

Department of Clinical Chemistry,

Laboratory Genetic Metabolic Diseases,

Academic Medical Center, University of Amsterdam,

Amsterdam, The Netherlands

M. Engelen · B. T. Poll-The

Department of Neurology, Academic Medical Center, University of Amsterdam,

Amsterdam, The Netherlands

M. Engelen $\cdot$ B. T. Poll-The $\cdot$ S. Kemp

Department of Pediatric Neurology/ Emma Children's Hospital, Academic Medical Center, University of Amsterdam,

Amsterdam, The Netherlands patients with hyperlipidaemia reduces VLCFA levels in X-ALD fibroblasts. Surprisingly, the VLCFA-lowering effect was independent of PPAR activation and not caused by the increase in either mitochondrial or peroxisomal fatty acid $\beta$-oxidation capacity. In fact, our results show that bezafibrate reduces VLCFA synthesis by decreasing the synthesis of C26:0 through a direct inhibition of fatty acid elongation activity. Taken together, our data indicate bezafibrate as a potential pharmacotherapeutic treatment for X-ALD. A clinical trial is currently ongoing to evaluate the effect in patients with X-ALD.

\footnotetext{
S. Fourcade $\cdot$ A. Pujol

Neurometabolic Diseases Laboratory,

The Bellvitge Institute of Biomedical Research (IDIBELL),

Center for Biomedical Research on Rare Diseases (CIBERER),

Barcelona, Spain

A. Pujol

ICREA (Institució Catalana de Recerca i Estudis Avançats),

Barcelona, Spain

S. Kemp $(\triangle)$

Department of Clinical Chemistry and Pediatric Neurology, Laboratory Genetic Metabolic Diseases,

Academic Medical Center,

Meibergdreef 9,

1105AZ Amsterdam, The Netherlands

e-mail: s.kemp@amc.uva.nl
} 


\section{Introduction}

X-linked adrenoleukodystrophy (X-ALD: OMIM 300100) is an inherited metabolic disorder characterized by impaired peroxisomal $\beta$-oxidation of very long-chain fatty acids (VLCFA; $\geq$ C22) and accumulation of VLCFA (mainly $\geq \mathrm{C} 26: 0$ ) in plasma and tissues of patients (Moser et al 2001). It is caused by mutations in the $A B C D 1$ gene (http://www.x-ald.nl), encoding a peroxisomal transmembrane protein named ALD protein (ALDP: OMIM 300371) (Mosser et al 1993). Clinically, X-ALD is characterized by a striking and unpredictable variation in phenotypic expression, ranging from the rapidly progressive childhood cerebral form (CCALD) to the more slowly progressive adult form adrenomyeloneuropathy (AMN) and variants without neurological involvement ("Addison-only" phenotype) (Moser et al 2001).

Experimental pharmacotherapy in X-ALD was aimed at normalizing VLCFA $\beta$-oxidation and VLCFA levels. Over the years several compounds have been investigated, such as Lorenzo's oil (Aubourg et al 1993; van Geel et al 1999), 4-phenylbutyrate (Kemp et al 1998), and lovastatin (Engelen et al 2010; Singh et al 1998). These treatments were shown to be either unpractical or ineffective in clinical trials and therefore other drugs are needed.

Fenofibrate (a PPAR- $\alpha$ agonist) was shown to induce expression of $A L D R(A b c d 2)$ in the liver of $A b c d 1^{-/-}$mice (Netik et al 1999). ALDRP is a functional homolog of ALDP (Kemp et al 1998). However, fenofibrate has no effect on $A b c d 2$ expression in the brain of $A b c d 1^{-/-}$mice, possibly because it is a substrate for the $M d r l$ transporter at the blood brain barrier and therefore does not penetrate into the brain very effectively (Berger et al 1999). For this reason, we investigated the effect of several other drugs known to activate PPAR on VLCFA metabolism in cultured skin fibroblasts from patients with X-ALD. The results described in this paper show that bezafibrate (BF), but not fenofibrate, clofibrate or other PPAR agonists, could reduce VLCFA in cultured fibroblasts from patients with X-ALD.

The VLCFA which accumulate in X-ALD, are partly absorbed from the diet (Kishimoto et al 1980), but mostly result from endogenous synthesis through elongation of long-chain fatty acids (Tsuji et al 1981). Recently, we identified ELOVL1 as the key enzyme responsible for the synthesis of VLCFA and demonstrated that knock-down of ELOVL1 resulted in lower VLCFA synthesis and reduced levels of VLCFA in cultured X-ALD fibroblasts (Ofman et al 2010). Hence, inhibiting fatty acid elongation (for example by inhibition of ELOVL1) by pharmacological means could be a potential treatment for X-ALD. Here, we show that BF lowers VLCFA in X-ALD fibroblasts by direct inhibition of fatty acid elongation.

\section{Materials and methods}

\section{Chemicals}

Deuterium-labeled palmitate-16,16,16- $\mathrm{D}_{3}$ acid $\left(\mathrm{D}_{3} \mathrm{C} 16: 0\right)$ was purchased from $\mathrm{CDN}$ isotopes (Pointe-Claire, Canada). A $12.5 \mathrm{mM}$ stock solution in dimethyl sulfoxide (DMSO) was prepared. BF, fenofibrate and clofibrate, WY14643, GW501516, and rosiglitazone were purchased from Sigma-Aldrich (St. Louis, MO, USA). Stock solutions in DMSO were made of $400 \mathrm{mM}$ (BF and clofibrate), $50 \mathrm{mM}$ (fenofibrate), $10 \mathrm{mM}$ (WY14643 and rosiglitazone), $500 \mu \mathrm{M}$ (GW501516). MK-886 was purchased from Cayman Chemical (Ann Arbor, MI, USA) and a stock solution of $50 \mathrm{mM}$ in DMSO was used. Prior to usage the stock solutions were vortex mixed and diluted in HAMF10 tissue culture medium to the final concentration. All chemicals used were of analytical grade.

Cell lines and cell culture

Primary human skin fibroblasts were obtained from X-ALD patients through the Neurology Outpatient Clinic of the Academic Medical Center. From each patient written informed consent was obtained. X-ALD diagnosis was confirmed by VLCFA and $A B C D 1$ mutation analysis. Control fibroblasts were from male anonymous volunteers. Cells from patients with a peroxisomal biogenesis disorder were obtained from the laboratory cell bank. Cells were grown in HAMF10 supplemented with $10 \%$ fetal calf serum, $2.5 \mathrm{mM}$ HEPES, $100 \mathrm{U} / \mathrm{ml}$ penicillin, $100 \mathrm{U} / \mathrm{ml}$ streptomycin and $2 \mathrm{mM}$ glutamine. Cells were used between passage numbers 6 and 20. Culture media were refreshed every 5 days.

Fatty acid synthesis

Synthesis of $\mathrm{D}_{3}$-VLCFA in intact cells was measured using $\mathrm{D}_{3}-\mathrm{C} 16: 0$. Assays were performed in triplicate. Cells were seeded at $40 \%$ confluency in T75 flasks. The next day, medium was replaced by fresh medium supplemented with $\mathrm{D}_{3}-\mathrm{C} 16: 0$ (dissolved in DMSO) at a final concentration of $50 \mu \mathrm{M}$. After $72 \mathrm{hr}$, cells were harvested and VLCFA analyzed as described (Valianpour et al 2003).

Measurement of fatty acid $\beta$-oxidation

Mitochondrial $\beta$-oxidation activity of intact fibroblasts was measured by quantifying the production of ${ }^{3} \mathrm{H}_{2} \mathrm{O}$ from $\left[9,10-{ }^{3} \mathrm{H}(\mathrm{N})\right]$ oleic acid as described previously (Moon and Rhead 1987). Peroxisomal $\beta$-oxidation activity of intact fibroblasts was measured using $\left[1-{ }^{14} \mathrm{C}\right]-26: 0$ as described previously (Wanders et al 1995). All measurements were performed in triplicate for each cell line. 
Immunofluorescence and counting of peroxisomes

Fibroblasts where grown on glass microscopy slides in 6-well plates with or without $400 \mu \mathrm{M}$ BF. Cells were fixed with paraformaldehyde and permeabilized with Triton X-100. Peroxisomes were visualized by catalase immunofluorescence microscopy as described previously (Kemp et al 1996). To count peroxisomes, we made images of immunofluorescencestained cells after focusing on the cell nucleus, and determined the peroxisome number per cell with the aid of a colony counter. For each cell line and condition, 10 cells were counted at random.

\section{Quantitative RT-PCR analysis}

ELOVL1, ELOVL4, ELOVL6, ACOX1, ABCD3 and CPT1a mRNA levels in control and X-ALD fibroblasts growing in log phase were determined as described (Engelen et al 2008), with primer sets presented in Table S1 (Supporting information Table S1).

\section{Purification of mouse liver microsomes}

Microsomes were isolated from livers from wild type and transgenic ELOVL1 over-expressing mice (Kemp et al manuscript in preparation) by differential centrifugation as described by Baudhuin et al (Baudhuin et al 1964), with minor modifications. Livers were washed with ice-cold homogenization buffer containing $250 \mathrm{mM}$ sucrose, $2 \mathrm{mM}$ EDTA, $2 \mathrm{mM}$ DTT and 5 mM MOPS (pH 7.4), minced and homogenized using a potter tissue grinder with Teflon pestle with 5 strokes at $500 \mathrm{rpm}$. A post-nuclear supernatant was produced by centrifugation at $600 \mathrm{~g}$ for $10 \mathrm{~min}$. The supernatant was centrifuged at 22,500 $\mathrm{g}$ for $10 \mathrm{~min}$ and the pellet was discarded. Next, the supernatant was centrifuged for $1 \mathrm{~h}$ at 100,000 $\mathrm{g}$ to obtain a microsomal fraction. The pellet was resuspended in homogenization buffer containing $10 \mathrm{mg} / \mathrm{mL}$ methyl- $\beta$-cyclodextrin and sonicated for 4 times 5 seconds at $8 \mathrm{~W}$ with a 1 minute interval. The microsomal membranes were collected by centrifugation at $100,000 \mathrm{~g}$ for $1 \mathrm{~h}$. Finally, the microsomes were resuspended in homogenization buffer and stored at $-80^{\circ} \mathrm{C}$ until further use. All steps were carried out at $4^{\circ} \mathrm{C}$. Protein concentrations were determined using BCA as described (Smith et al 1985).

Fatty acid elongation assay

The fatty acid elongation assay was carried out using a method adapted from Nagi et al (Nagi et al 1989). The reaction mixture contained $50 \mathrm{mM}$ potassium phosphate buffer $(\mathrm{pH}$ 6.5), $10 \mathrm{mg} / \mathrm{mL} \alpha$-cyclodextrin, $1 \mathrm{mM}$ NADPH, $5 \mu \mathrm{M}$ rotenone, $60 \mu \mathrm{M}\left[2-{ }^{14} \mathrm{C}\right]$ malonyl-CoA (6.5 dpm/pmol) (American Radiolabeled Chemicals, St Louis, MO) and $20 \mu \mathrm{M}$ C16:0-CoA or $20 \mu \mathrm{M}$ C22:0-CoA (Avanti Polar Lipids, Alabaster, AL), in a total volume of $200 \mu \mathrm{L}$. The
Fig. 1 Only BF, and not other drugs from the fibrate class, reduces C26:0 in X-ALD fibroblasts. (a) $\mathrm{C} 26: 0$ levels in 3 X-ALD cell lines cultured for 7 days without drugs (black bar), in the presence of BF (gray bar), fenofibrate (white bar), or clofibrate (hatched bar). (b) C26:0 levels in $3 \mathrm{X}$-ALD cell lines cultured for 7 days without drugs (black bar), in the presence of BF (gray bar), agonists of PPAR- $\alpha$ (WY 14643), PPAR- $\beta$ (GW 501516) and PPAR- $\gamma$ (rosiglitazon), or with different combinations of PPAR agonists (white bars). (c) C26:0 levels in 8 control (white bar) and 8 X-ALD cell lines cultured without (black bar) or with $400 \mu \mathrm{M} \mathrm{BF}$ (gray bars) for up to 3 weeks. Fatty acid levels are in $\mathrm{nmol} / \mathrm{mg}$ protein. Data are mean \pm SD. $*=\mathrm{p}<0.05$,

$* *=P<0.01, * * *=P<0.001$ by ANOVA followed by Dunnett's multiple comparison test compared with untreated X-ALD cells
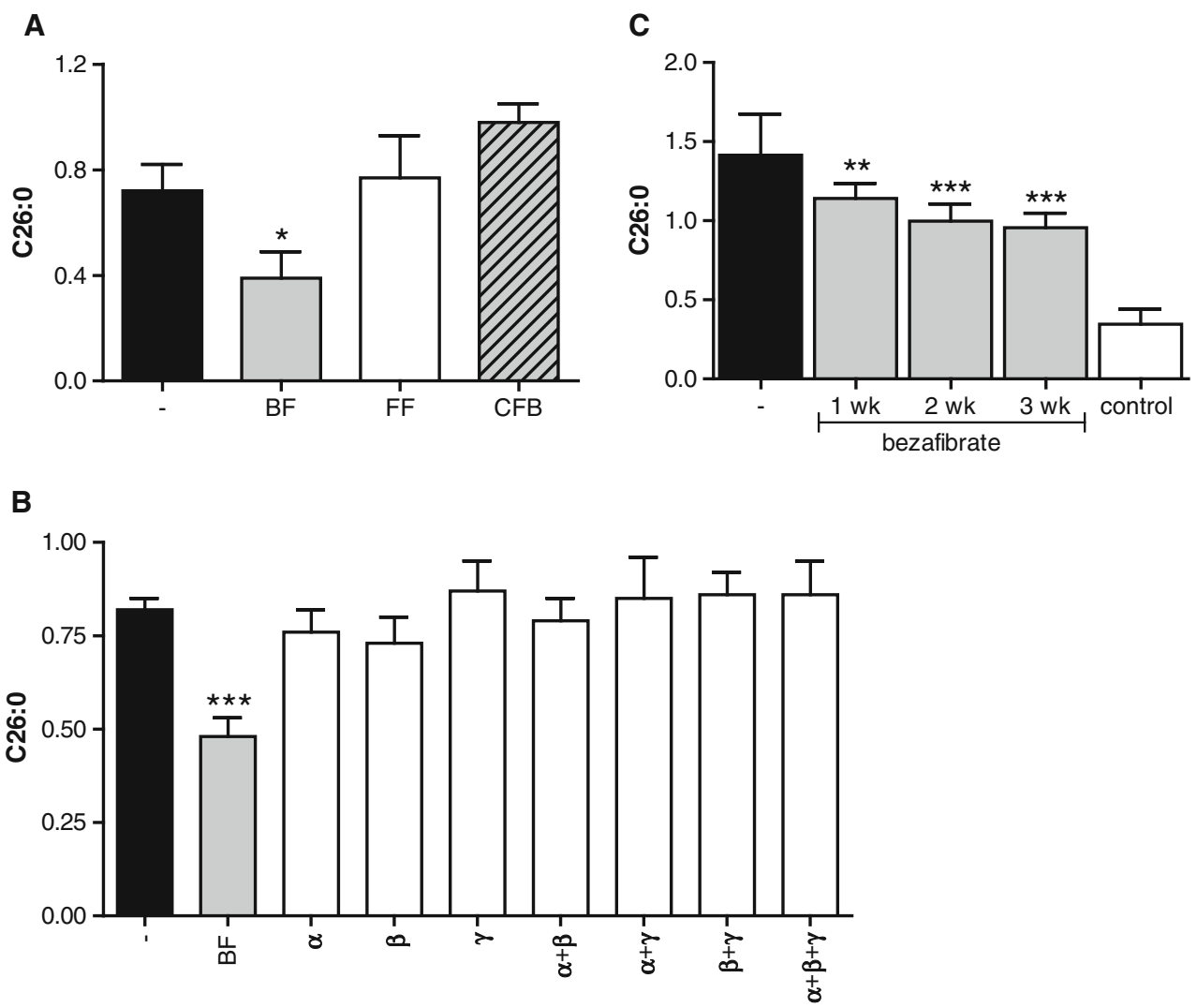
reaction was started by adding $100 \mu \mathrm{g}$ protein of the microsomal fraction and allowed to proceed for $30 \mathrm{~min}$ at $37^{\circ} \mathrm{C}$. $\mathrm{NADPH}$ dependency was tested by performing the reaction without NADPH. Reactions were carried out with or without $\mathrm{BF}$ or BF-CoA $(100-400 \mu \mathrm{M})$. The reaction was stopped by adding $200 \mu \mathrm{L} 5 \mathrm{M} \mathrm{KOH}$ in $10 \%$ methanol, saponified at $65^{\circ}$ $\mathrm{C}$ for $1 \mathrm{~h}$ and acidified by adding $200 \mu \mathrm{L} 5 \mathrm{~N} \mathrm{HCl}$ and $200 \mu \mathrm{L}$ $96 \%$ ethanol. Fatty acids were extracted three times with $1 \mathrm{~mL}$ hexane and the hexane phases were collected in a scintillation vial to which $10 \mathrm{~mL}$ scintillation cocktail (Ultima-Gold, Perkin Elmer) was added and radioactivity counted.

\section{Synthesis of bezafibroyl-CoA (BF-CoA)}

BF-CoA was synthesized by a method adapted from Rasmussen et al (Rasmussen et al 1990). Dichloromethane (DCM) and tetrahydrofuran (THF) (Merck) were dried with molecular sieve deperox (Fluka). Triethylamine and ethylchloroformate (Merck) were diluted to $1 \mathrm{M}$ with dry DCM. The reaction contained $36 \mu \mathrm{mol} \mathrm{BF}$ dissolved in $1.4 \mathrm{~mL} \mathrm{DCM} /$ THF (5:2) and $40 \mu \mathrm{L} 1 \mathrm{M}$ triethylamine. The reaction mixture was incubated at room temperature for $10 \mathrm{~min}$ under constant stirring and under an atmosphere of nitrogen. After $10 \mathrm{~min}, 40$ $\mu \mathrm{L} 1 \mathrm{M}$ ethylchloroformate was added and the incubation was continued for $45 \mathrm{~min}$. After the incubation, the mixture was dried under nitrogen and dissolved in $0.5 \mathrm{~mL}$ tert-butanol. Next, $40 \mu \mathrm{mol}$ CoA trilithium salt (Sigma-Aldrich) dissolved in $0.5 \mathrm{~mL} 0.4 \mathrm{M}$ potassium bicarbonate was added and the sample was incubated for $30 \mathrm{~min}$ at room temperature. The reaction was stopped by adding $100 \mu \mathrm{L} 0.1 \mathrm{~N} \mathrm{HCl}$. BF-CoA was purified using a $\mathrm{C} 18$ solid phase extraction column (JTBaker). The column was eluted by a gradient of acetonitrile and $40 \mathrm{mM}$ ammonium acetate, starting with $10 \%$ acetonitrile and $90 \% 40 \mathrm{mM}$ ammonium acetate increasing to $50 \%$ acetonitrile and 50\% $40 \mathrm{mM}$ ammonium acetate. Acetonitrile was evaporated and purity was checked by HPLC. BF-CoA was quantified using 5,5'-dithiobis-(2-nitrobenzoic) acid (DTNB) (Sigma-Aldrich). The method used was an adaptation of that of Ellman (Ellman 1959). BF-CoA was diluted in $20 \mu \mathrm{M}$ MES buffer $\mathrm{pH} 6.0$, an equal volume of $2 \mathrm{M} \mathrm{NaOH}$ was added and the sample was incubated for $30 \mathrm{~min}$ at $50^{\circ} \mathrm{C}$. The reaction was neutralized with $2 \mathrm{M} \mathrm{HCl}$. Absorbance at $412 \mathrm{~nm}$ was measured and the concentration was determined using a calibration curve of CoA trilithium salt (Sigma-Aldrich).

\section{Results}

Effect of BF on endogenous VLCFA levels and de novo C26:0 synthesis in fibroblasts from patients with X-ALD

We tested the effect of several drugs from the fibrate class, but found that only BF reduces C26:0 levels, both fenofibrate and clofibrate being ineffective (Fig. 1a). BF is a PPAR panagonist activating all three PPARs. To determine whether the effect of $\mathrm{BF}$ on $\mathrm{C} 26: 0$ levels is mediated by activation of either $\operatorname{PPAR} \alpha, \operatorname{PPAR} \beta / \delta$ or PPAR $\gamma, \mathrm{X}-\mathrm{ALD}$ fibroblasts were incubated with the PPAR $\alpha$ ligand WY14643, PPAR $\beta / \delta$ ligand GW501516, or the PPAR $\gamma$ ligand rosiglitazone either alone or in all possible combinations. Only treatment with BF reduced C26:0 levels by about $30 \%$ after 7 days (Fig. 1b).

To examine if longer incubations with BF would result in a further decrease of C26:0 levels, we cultured X-ALD fibroblasts for up to 21 days. This resulted in a small additional decrease of $\mathrm{C} 26: 0$ of $10 \%$ after 14 days and $15 \%$ after 21 days, respectively (Fig. 1c). No signs of

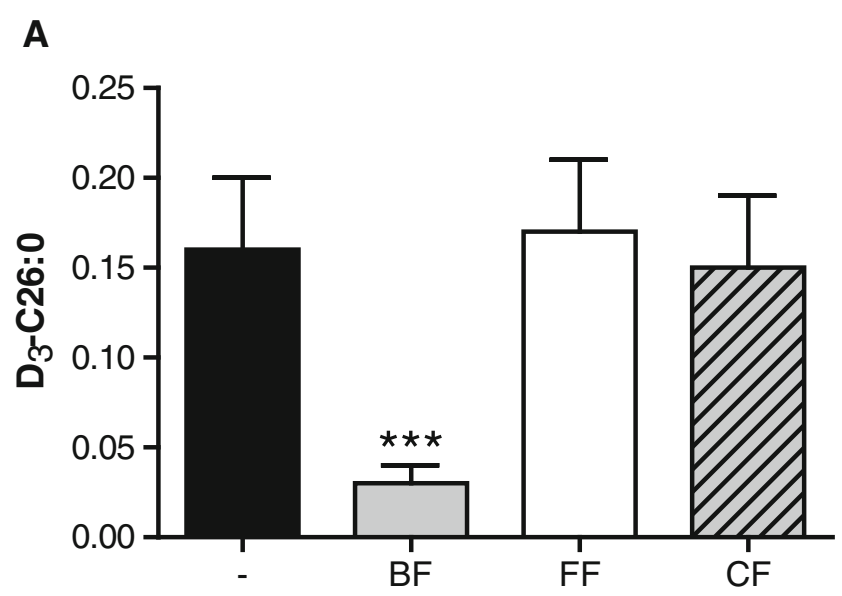

B

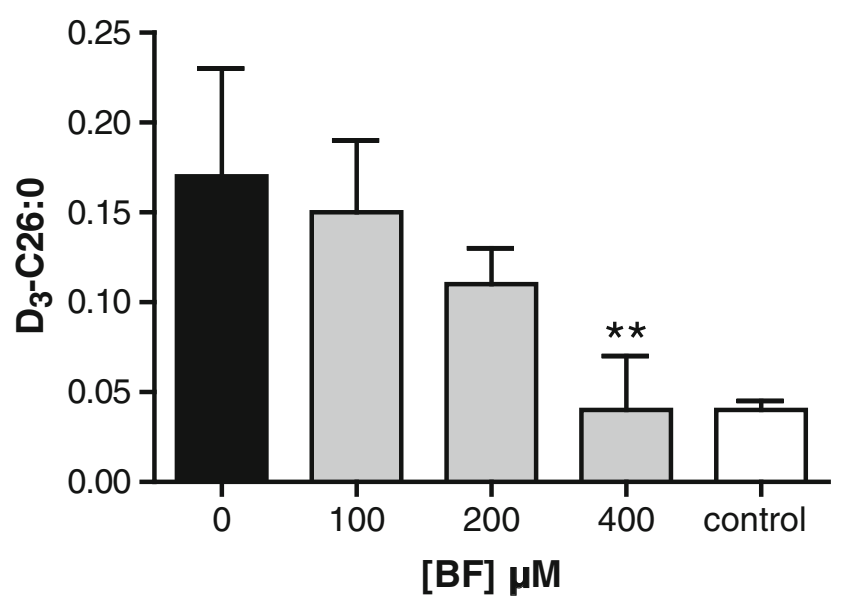

Fig. $2 \mathrm{BF}$, but not other fibrates, inhibits $\mathrm{D}_{3}-\mathrm{C} 26: 0$ synthesis. (a) $\mathrm{D}_{3^{-}}$ C26:0 synthesis from $D_{3}-C 16: 0$ in 5 X-ALD cell lines cultured for 3 days without drugs (black bar) and in the presence of BF (gray bar), fenofibrate (FF, white bar), or clofibrate (CF, hatched bar). (b) $\mathrm{D}_{3^{-}}$ C26:0 analysis from $\mathrm{D}_{3}-\mathrm{C} 16: 0$ in 4 control (white bar) and 4 X-ALD cell lines cultured for 3 days without (black bar) or with increasing concentrations of BF (gray bars). Fatty acid levels are in nmol $/ \mathrm{mg}$ protein. Data are mean \pm SD. $* *=P<0.01, * * *=P<0.001$ by ANOVA followed by Dunnett's multiple comparison test compared with untreated X-ALD cells 
Fig. 3 Effect of BF on mitochondrial and peroxisomal $\beta$-oxidation and peroxisome number. Measurement of (a) C16:0 $\beta$-oxidation and (b) $\mathrm{C} 26: 0 \beta$-oxidation activity in 5 control cell lines (white bar) and 5 X-ALD cell lines cultured without (black bar) or with $400 \mu \mathrm{M}$ BF (gray bar) for 7 days. Activities are in pmol/ $\mathrm{mg} /$ hour. Data are mean $\pm \mathrm{SD}$. $*=P<0.05$ and $* * *=P<0.001$ by ANOVA followed by Dunnett's multiple comparison test compared with untreated XALD cells. (c) Peroxisome number in $10 \mathrm{X}$-ALD cell lines cultured without (white bar) or with $400 \mu \mathrm{M}$ BF (gray bar) for 3 days. Mean and quartiles are indicated, the error bars represent the range
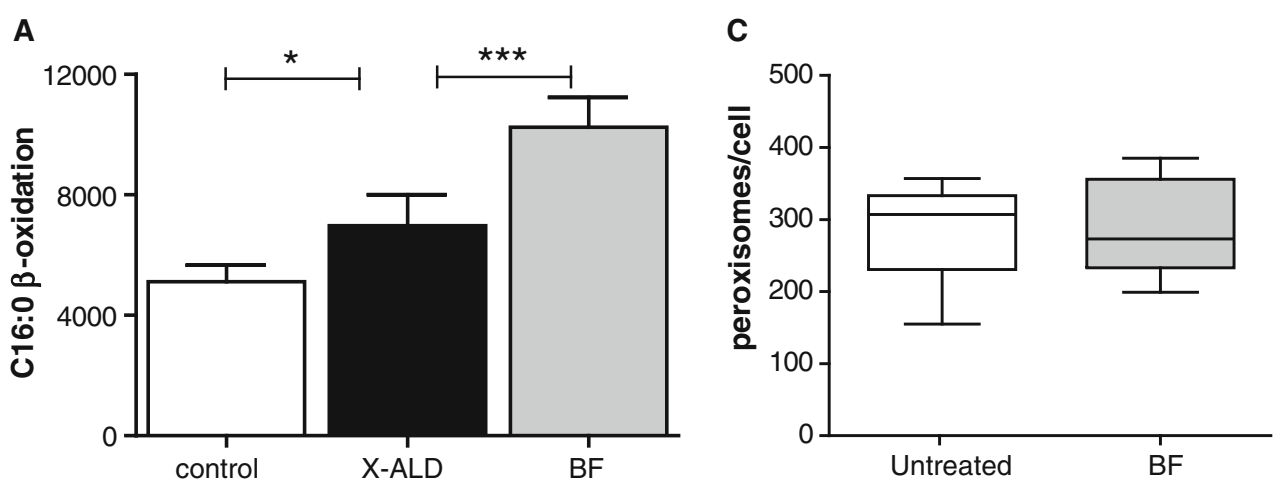

cytotoxicity or impaired growth as determined by the MTS cell proliferation assay (CellTiter $96^{\circledR}$ Aqueous One Solution Cell Proliferation Assay) were observed (data not shown). Previously, we validated the use of stable-isotope labeled fatty acids to study VLCFA de novo synthesis in whole cells and demonstrated that the synthesis of $\mathrm{D}_{3}-\mathrm{C} 26: 0$ from $\mathrm{D}_{3}-\mathrm{C} 16: 0$ is elevated in X-ALD fibroblasts (Ofman et al 2010). Earlier work has shown that BF is a potent inhibitor of long-chain fatty acid elongation, while clofibrate is not (Sanchez et al 1993; Vazquez et al 1995). Therefore, we tested the effect of BF, clofibrate and fenofibrate on C26:0 de novo synthesis. Of all fibrates tested, only $\mathrm{BF}$ affected the de novo $\mathrm{D}_{3}$-C26:0 synthesis (Fig. $2 \mathrm{a}$ ). $\mathrm{BF}$ reduced $\mathrm{D}_{3}-\mathrm{C} 26: 0$ synthesis in a concentration-dependent manner. At $400 \mu \mathrm{M}$ $\mathrm{BF}$, the synthesis of $\mathrm{D}_{3}-\mathrm{C} 26: 0$ was reduced by $75 \%$ compared with untreated X-ALD fibroblasts (Fig. 2b). At this concentration, there was no difference in the amount of newly synthesized $\mathrm{D}_{3}-\mathrm{C} 26: 0$ between X-ALD and control cells. We performed further experiments with $\mathrm{BF}$, and not the other fibrates or PPAR agonists, to identify by which mechanism $\mathrm{BF}$ reduces $\mathrm{C} 26: 0$ levels in fibroblasts from patients with X-ALD.

The effect of BF on $\mathrm{C} 26: 0$ levels is independent of induction of mitochondrial or peroxisomal $\beta$-oxidation

$\mathrm{BF}$ and PPAR ligands in general are known to induce mitochondrial and peroxisomal $\beta$-oxidation (Bonnefont et al 2009; Cabrero et al 2001; Islinger et al 2007; Pyper et al
2010). The effect of BF treatment on the rate of mitochondrial and peroxisomal fatty acid $\beta$-oxidation was determined in X-ALD fibroblasts. Exposure of X-ALD fibroblasts to BF caused a 50\% increase in C16:0 $\beta$-oxidation (Fig. 3a), and a $35 \%$ increase in $\mathrm{C} 26: 0 \beta$-oxidation (Fig. $3 \mathrm{~b}$ ). Since it is known that fibrates induce peroxisome proliferation in rodents (Islinger et al 2007), we determined the amount of peroxisomes in X-ALD fibroblasts incubated for 10 days

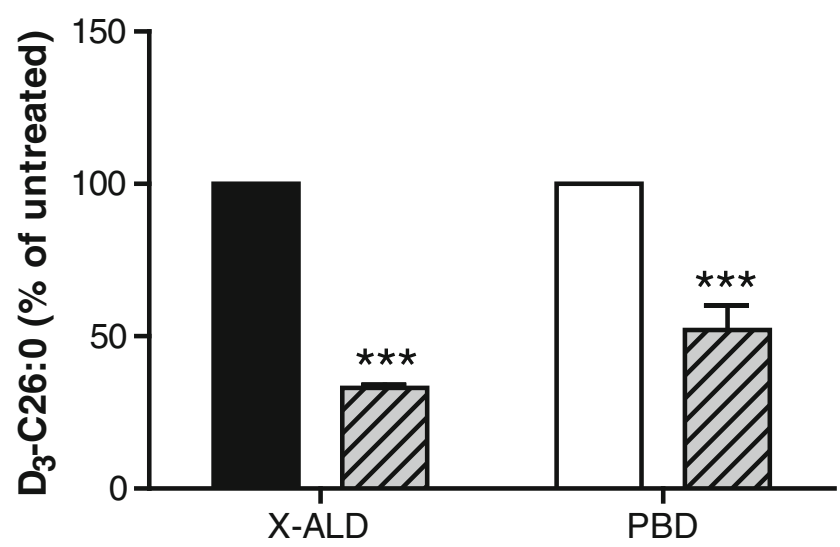

Fig. $4 \mathrm{C} 26: 0$ reduction by $\mathrm{BF}$ is not mediated by increased peroxisomal $\beta$-oxidation. Analysis of de novo $\mathrm{D}_{3}$-C26:0 synthesis in $3 \mathrm{X}$ ALD (black bar) cell lines and 3 cell lines from patients with a peroxisomal biogenesis disorder ( $\mathrm{PBD}$, white bar). Cells were incubated with $50 \mu \mathrm{M} \mathrm{D}_{3}-\mathrm{C} 16: 0$ for 3 days without or with $400 \mu \mathrm{M}$ BF (crosshatched bars). Fatty acid levels are in $\mathrm{nmol} / \mathrm{mg}$ protein. Data are mean \pm SD. ${ }^{* * *}=p<0.001$ by student's unpaired $t$-test 
with BF. We did not find any evidence for an effect of BF treatment on peroxisome abundance (Fig. 3c).

$\mathrm{BF}$ reduced $\mathrm{C} 26: 0$ levels in cultured X-ALD fibroblasts, while other drugs of the fibrate class or other synthetic PPAR agonists could not. This strongly suggested that the effect of BF on VLCFA levels is not dependent solely on induction of mitochondrial or peroxisomal $\beta$-oxidation, since the other compounds should have been effective as well then (Blaauboer et al 1990; Kemp et al 2011). BF treatment resulted in a $35 \%$ increase in the peroxisomal C26:0 $\beta$-oxidation capacity (Fig. $3 b$ ). To test if the induction of peroxisomal $\beta$-oxidation by $\mathrm{BF}$ caused the reduction in $\mathrm{C} 26: 0$ levels, we incubated fibroblasts from patients with

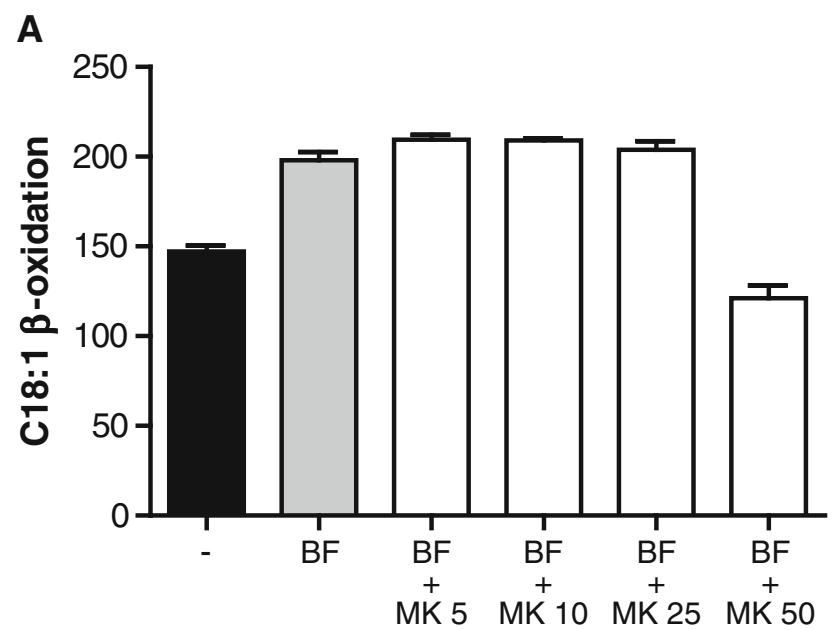

B

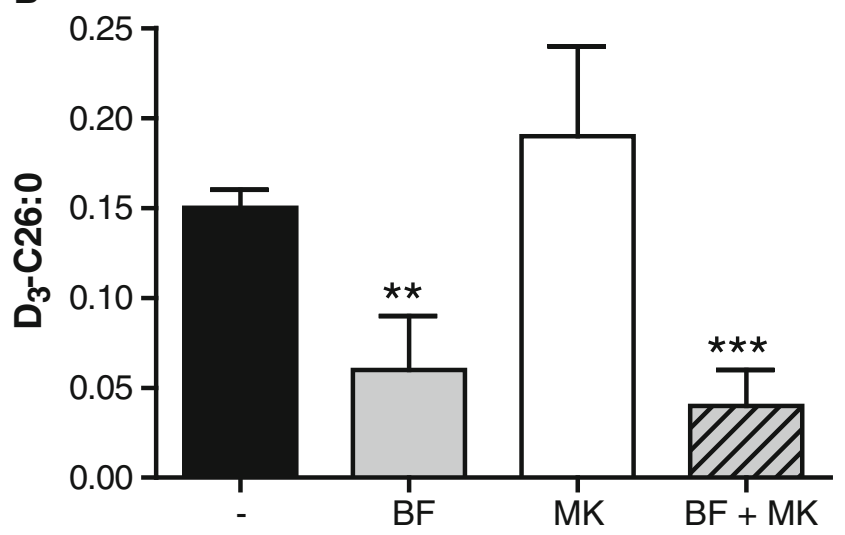

Fig. $5 \mathrm{C} 26: 0$ reduction by $\mathrm{BF}$ is not mediated by increased mitochondrial $\beta$-oxidation. (a) $\mathrm{C} 18: 1 \beta$-oxidation in $3 \mathrm{X}$-ALD cell lines cultured without (black bar), or for 48 hours with $400 \mu \mathrm{M}$ BF (gray bar), or with $\mathrm{BF}$ and increasing concentrations of MK-886 (white bars). Activities are in $\mathrm{pmol} / \mathrm{mg} /$ hour. Data are mean $\pm \mathrm{SD}$. (b) De novo $\mathrm{D}_{3}$ C26:0 synthesis in 4 X-ALD cell lines incubated with $50 \mu \mathrm{M} \mathrm{D}_{3}-\mathrm{C} 16: 0$ without (black bar) or with $400 \mu \mathrm{M}$ BF (gray bar), $50 \mu \mathrm{M}$ MK-886 (white bar), or $400 \mu \mathrm{M} \mathrm{BF}$ and $50 \mu \mathrm{M}$ MK-886 (cross-hatched bar). Fatty acid levels are in $\mathrm{nmol} / \mathrm{mg}$ protein. Data are mean \pm SD. $* *=p<$ $0.01 ; * * *=p<0.001$ by ANOVA followed by Dunnett's multiple comparison test compared with untreated X-ALD cells a peroxisomal biogenesis disorder (PEX1, PEX6 and PEX26) with $\mathrm{BF}$ and measured the effect on $\mathrm{D}_{3}-\mathrm{C} 26: 0$ de novo synthesis. The synthesis of $\mathrm{D}_{3}-\mathrm{C} 26: 0$ from $\mathrm{D}_{3}-\mathrm{C} 16: 0$ was reduced roughly $50 \%$ in peroxisome-deficient fibroblasts incubated with BF (Fig. 4). This clearly indicated that the effect of $\mathrm{BF}$ on $\mathrm{D}_{3}-\mathrm{C} 26: 0$ synthesis and $\mathrm{C} 26: 0$ levels is only partially mediated by an induction of the peroxisomal $\beta$-oxidation capacity.

To investigate whether the induction of mitochondrial $\beta$ oxidation by $\mathrm{BF}$ is responsible for the reduction in $\mathrm{C} 26: 0$, we used the PPAR $\alpha$ inhibitor MK-886 (Kehrer et al 2001). The induction of mitochondrial $\beta$-oxidation by $\mathrm{BF}$ in $\mathrm{X}$ ALD fibroblasts could be reversed with $50 \mu \mathrm{M}$ MK-886, suggesting that PPAR $\alpha$ activation is completely blocked at this concentration (Fig. 5a). Next, we measured $\mathrm{D}_{3}-\mathrm{C} 26: 0$ de novo synthesis in X-ALD fibroblasts incubated with BF in the presence of MK-886. Addition of MK-886 did not affect the reduction of $\mathrm{D}_{3}-\mathrm{C} 26: 0$ synthesis (Fig. 5b). Combined, these data are highly suggestive that the reduction of C26:0 levels by BF is PPAR independent and can not be explained by the increase in mitochondrial $\beta$-oxidation capacity and only partially by the increase in peroxisomal $\beta$ oxidation capacity. This suggests that $\mathrm{BF}$ inhibits the formation of $\mathrm{D}_{3}-\mathrm{C} 26: 0$.

\section{BF directly inhibits fatty acid elongation}

The amount of $\mathrm{D}_{3}-\mathrm{C} 26: 0$ present is the net result of the elongation of $\mathrm{D}_{3}-\mathrm{C} 16: 0$ to $\mathrm{D}_{3}-\mathrm{C} 26: 0$ by ELOVL6 and ELOVL1, respectively (Ofman et al 2010) and the degradation of $\mathrm{D}_{3}$-C26:0 by peroxisomal $\beta$-oxidation. The inhibitory effect of $\mathrm{BF}$ on the formation of $\mathrm{D}_{3}-\mathrm{C} 26: 0$ could either be indirect by affecting gene expression or direct by inhibition of key enzymes involved in VLCFA de novo synthesis (Ofman et al 2010). To investigate the effect of BF on gene expression levels of several key enzymes involved in VLCFA

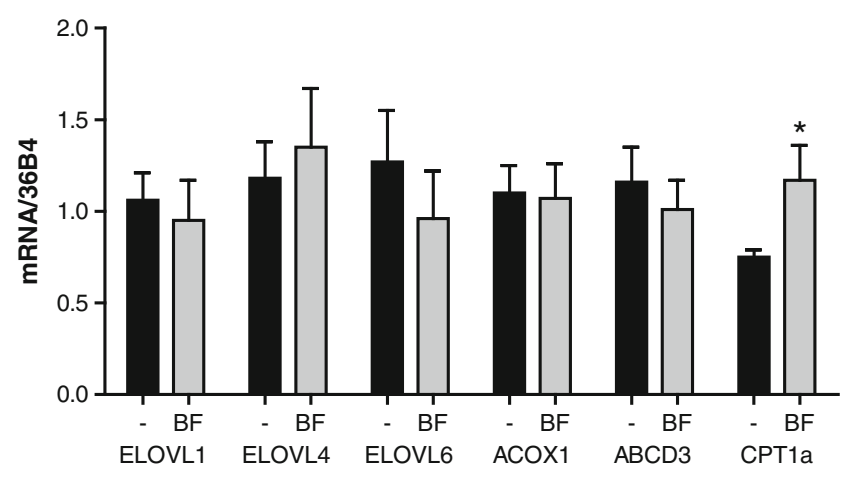

Fig. 6 Effect of BF on gene expression of genes involved in fatty acid metabolism. Expression levels of genes involved in fatty acid metabolism in 3 X-ALD cell lines cultured without (black bars) or with $400 \mu \mathrm{M}$ BF (gray bars) for 48 hours were analyzed by quantitative PCR. Data are mean \pm SD. $*=p<0.05$ by student's unpaired $t$-test 
synthesis, peroxisomal $\beta$-oxidation and mitochondrial $\beta$ oxidation we performed quantitative RT-PCR. As shown in Fig. 6, there is only a small reduction in the mRNA levels of ELOVL6, which is not statistically significant. There was no increase in the expression of $A C O X 1$. This is in line with previously published data (Blaauboer et al 1990) and our own data (Fig. 3c) showing that there is no peroxisome proliferation, at least in cultured human cells, treated with fibrates, in contrast to rodents (Blaauboer et al 1990). BF did not induce the expression of $A B C D 3$. Induction of this gene was detected in mice treated with fenofibrate, and considered to be the mechanism by which fibrates might be useful in correcting the metabolic defect in X-ALD (Berger et al 1999; Netik et al 1999). Our data show that induction of $A B C D 3$ did not occur in human X-ALD fibroblasts upon exposure to BF. In line with previous data (Djouadi et al 2005), BF treatment resulted in increased expression of CPTIa.

The previous experiments suggested that the effect of $\mathrm{BF}$ on $\mathrm{C} 26: 0$ levels and C26:0 synthesis could be mediated by a direct inhibiting effect on fatty acid elongation. VLCFA are synthesized by the concerted action of ELOVL6 and ELOVL1 (Ofman et al 2010). ELOVL6 elongates $\mathrm{C} 16: 0$ to $\mathrm{C} 22: 0$ and ELOVL1 elongates C22:0 to $\mathrm{C} 26: 0$. We measured the effect of free $\mathrm{BF}$ and $\mathrm{BF}$ esterified to coenzyme CoA (BF-CoA) on C16:0-CoA and $\mathrm{C} 22: 0-\mathrm{CoA}$ elongation. In the elongation assay $\mathrm{BF}$ had no effect. However, BF-CoA inhibited the chain elongation activity of both $\mathrm{C} 16: 0$ and $\mathrm{C} 22: 0$ in a concentration dependent manner (Fig. 7). Both fenofibrate and clofibrate did not inhibit $\mathrm{C} 22: 0-\mathrm{CoA}$ elongation (Fig. 7b). These data demonstrate that BF-CoA lowers C26:0 levels by direct inhibition of fatty acid chain elongation.

\section{Discussion}

Synthetic PPAR alpha ligands, like fibrates, are potentially interesting compounds to investigate as therapeutic agents in X-ALD because they are known to activate mitochondrial and peroxisomal fatty acid $\beta$-oxidation (Vazquez et al 2001). They might therefore reduce VLCFA accumulation by increasing VLCFA degradation. Indeed, Brown and colleagues demonstrated that treatment of two CCALD patients with clofibrate resulted in a reduction in VLCFA (Brown et al 1982). However, this reduction was not sustained. More recent experiments showed that fenofibrate induced expression of both ALDRP (ABCD2) and PMP70 (ABCD3) in the liver of $A b c d 1$-deficient mice, but not in brain (Berger et al 1999). In $M d r 1^{-/}$knockout mice induction of $A L D R P$ and PMP70 in brain did occur, suggesting that fenofibrate is indeed cleared from the brain by Mdr1 (Berger et al 1999). These studies, however, did not report the effect on VLCFA levels in tissues. In this paper, we studied the effect of several classical fibrates and other synthetic PPAR ligands in a cell model for X-ALD, and show that BF but not the other fibrates reduced endogenous C26:0 levels. This C26:0 reducing effect could not be mimicked by other PPARligands which means that the effect is PPAR independent. $\mathrm{BF}$ has been demonstrated to induce mitochondrial $\beta$ oxidation (Bonnefont et al 2009; Djouadi et al 2005). Blocking the induction of mitochondrial $\beta$-oxidation with MK-886 did not prevent the reduction of $\mathrm{D}_{3}-\mathrm{C} 26: 0$ de novo synthesis in X-ALD fibroblast by BF. We also showed that the peroxisomal $\mathrm{C} 26: 0 \beta$-oxidation capacity in X-ALD skin fibroblasts increased with $35 \%$ upon treatment with $\mathrm{BF}$. However this does not seem to be the only mechanism of reduction of VLCFA in fibroblasts incubated with BF,
Fig. 7 BF inhibits fatty acid elongation. (a) Fatty acid elongation activity of C16:0CoA (a substrate of ELOVL6) in the presence of an increasing concentration of BF (o) and BF$\mathrm{CoA}(\bullet)$. (b) Fatty acid elongation activity of $\mathrm{C} 22: 0-\mathrm{CoA}$ (a substrate of ELOVL1) in the presence of an increasing concentration of BF (o) and BF$\mathrm{CoA}(\bullet)$ or with an increasing concentration of fenofibrate $(\mathrm{FF}, \square)$ or clofibrate $(\mathrm{CF}, \Delta)$. Fatty acid elongation activity was measured using $20 \mu \mathrm{M}$ C16:0-CoA or C22:0-CoA as substrate with concentrations of the inhibitor up to $400 \mu \mathrm{M}$. Error bars represent the standard deviation

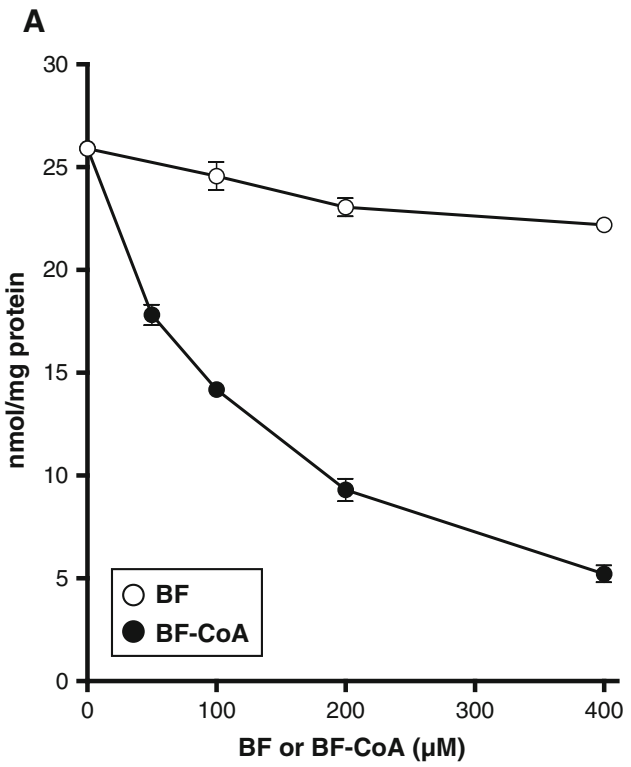

B

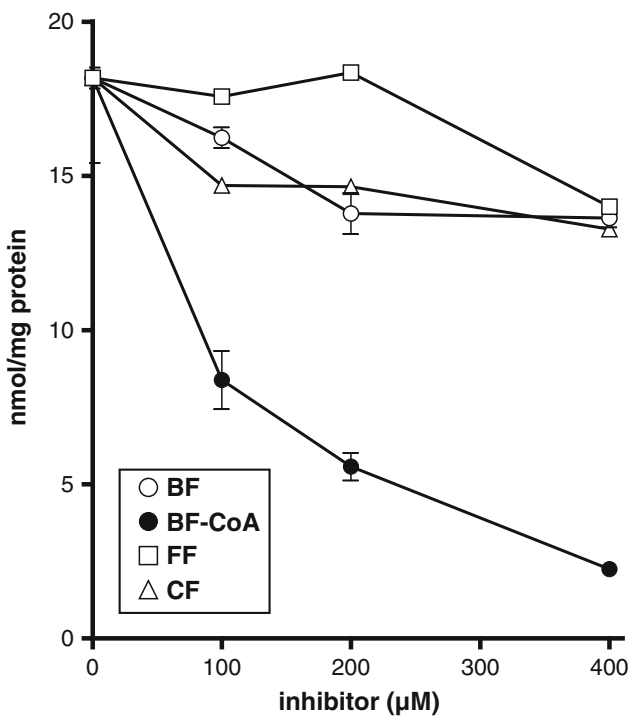


because in cells from patients with a peroxisome biogenesis disorder in which peroxisomal $\beta$-oxidation is completely deficient, $\mathrm{BF}$ lowered $\mathrm{D}_{3}$-C26:0 levels as well. This strongly suggested that $\mathrm{BF}$ might reduce $\mathrm{C} 26: 0$ levels primarily by inhibiting $\mathrm{C} 26: 0$ synthesis. To test this we measured de novo synthesis of $\mathrm{D}_{3}-\mathrm{C} 26: 0$ from $\mathrm{D}_{3}-\mathrm{C} 16: 0$ in X-ALD fibroblasts. $\mathrm{BF}$ indeed decreased de novo synthesis of $\mathrm{D}_{3}-\mathrm{C} 26: 0$ in a concentration-dependent manner. At a concentration of $400 \mu \mathrm{M} \mathrm{BF}, \mathrm{D}_{3}-\mathrm{C} 26: 0$ levels in X-ALD fibroblasts were at the level of control fibroblasts. The rate limiting enzymes involved in synthesis of $\mathrm{C} 26: 0$ from C16:0 are ELOVL6 (elongation of C16:0 to C22:0) and ELOVL1 (elongation of C22:0 to C26:0) (Kemp and Wanders 2010; Ofman et al 2010). By qPCR we showed that expression levels of these enzymes are not affected in fibroblasts incubated with BF suggesting a direct inhibition of VLCFA synthesis. Previously using rat liver microsomes other investigators showed that BF inhibits palmitoyl-CoA (C16:0-CoA) elongation in an in vitro assay (Sanchez et al 1993; Vazquez et al 1995). We used purified microsomes from wild type and ELOVL1 over-expressing mice to test the effect of BF on elongation of both long-chain fatty acids (LCFA) and VLCFA. Our results demonstrate that BF-CoA, but not free $\mathrm{BF}$, is a potent inhibitor of both LCFA and VLCFA elongation. It should be noted, however, that these results do not allow us to demonstrate at which level BF inhibits VLCFA synthesis. Fatty acid elongation requires four sequential reaction steps: (i) condensation between the fatty acyl-CoA and malonyl-CoA to form 3-ketoacyl-CoA; (ii) reduction using NADPH to form 3-hydroxyacyl-CoA; (iii) dehydration to trans-2-enoyl-CoA; and (iv) reduction to fully elongated fatty acyl-CoA. The initial condensation reaction is catalyzed by the enzyme referred to as "elongation of very long-chain fatty acids" (ELOVL) and is considered to be rate limiting (Cinti et al 1992). While seven elongases have been identified in mammals (designated ELOVL1ELOVL7)(Jakobsson et al 2006), only a single enzyme has been identified yet for the subsequent reaction step (Jakobsson et al 2006). Identification of the specific enzyme(s) affected by BF is not a trivial thing and requires detailed analysis of all enzymes involved, including: 3ketoacyl-CoA reductase (HSD17B12), 3-hydroxyacyl dehydratase (HACD3) and the trans-2,3-enoyl-CoA reductase (TECR). This will be the subject of future studies.

\section{Concluding remarks}

The work described in the paper shows that inhibition of VLCFA synthesis by pharmacological means could be a feasible treatment option for X-ALD. BF is a good candidate for this approach. BF lowers the levels of $\mathrm{C} 26: 0$ by a direct inhibition of the synthesis. Mouse studies to evaluate the in vivo effect of BF treatment on VLCFA in X-ALD mice would be interesting; however, the effect of fibrates is quite different in rodents and humans. Watanabe et al demonstrated that rats and mice are unusable as a model system (for primates) to the study the effect of BF (Watanabe et al 1989). BF has a proven safety profile for (long-term) use in humans. With a daily dose of $200 \mathrm{mg}$ of BF peak plasma levels of $50 \mu \mathrm{M}$ can be reached, with a maximum daily dose of $800 \mathrm{mg}$ of $\mathrm{BF}$ therapeutic levels might be reached in plasma (Miller and Spence 1998). A small scale proof of principle clinical trial is currently ongoing to evaluate the effect in X-ALD patients.

Acknowledgements We thank Femke Stet, Petra Mooyer, and Henk van Lenthe for expert technical assistance. This research was supported by the Netherlands Organization for Scientific Research (VIDI-grant number 91786328), the European Union Framework Programme 7 (grant number LeukoTreat 241622). The funders had no role in study design, data collection and analysis, decision to publish, or preparation of the manuscript.

Conflict of interest This research was supported by the Netherlands Organization for Scientific Research (VIDI-grant number 91786328), the European Union Framework Programme 7 (grant number LeukoTreat 241622). The funders had no role in study design, data collection and analysis, decision to publish, or preparation of the manuscript.

Open Access This article is distributed under the terms of the Creative Commons Attribution License which permits any use, distribution, and reproduction in any medium, provided the original author(s) and the source are credited.

\section{References}

Aubourg P, Adamsbaum C, Lavallard-Rousseau MC et al (1993) A two-year trial of oleic and erucic acids ("Lorenzo's oil") as treatment for adrenomyeloneuropathy. N Engl J Med 329:745-752

Baudhuin P, Beaufay H, Rahman-Li Y et al (1964) Tissue fractionation studies. 17. Intracellular distribution of monoamine oxidase, aspartate aminotransferase, alanine aminotransferase, D-amino acid oxidase and catalase in rat-liver tissue. Biochem J 92:179-184

Berger J, Albet S, Bentejac M et al (1999) The four murine peroxisomal ABC-transporter genes differ in constitutive, inducible and developmental expression. Eur J Biochem 265:719-727

Blaauboer BJ, van Holsteijn CW, Bleumink R et al (1990) The effect of beclobric acid and clofibric acid on peroxisomal beta-oxidation and peroxisome proliferation in primary cultures of rat, monkey and human hepatocytes. Biochem Pharmacol 40:521-528

Bonnefont JP, Bastin J, Behin A, Djouadi F (2009) Bezafibrate for an inborn mitochondrial beta-oxidation defect. N Engl J Med 360:838-840

Brown FR, Van Duyn MA, Moser AB et al (1982) Adrenoleukodystrophy: effects of dietary restriction of very long chain fatty acids and of administration of carnitine and clofibrate on clinical status and plasma fatty acids. Johns Hopkins Med J 151:164-172

Cabrero A, Alegret M, Sanchez RM, Adzet T, Laguna JC, Vazquez M (2001) Bezafibrate reduces mRNA levels of adipocyte markers 
and increases fatty acid oxidation in primary culture of adipocytes. Diabetes 50:1883-1890

Cinti DL, Cook L, Nagi MN, Suneja SK (1992) The fatty acid chain elongation system of mammalian endoplasmic reticulum. Progress in Lipid Research 31:1-51

Djouadi F, Aubey F, Schlemmer D et al (2005) Bezafibrate increases very-long-chain acyl-CoA dehydrogenase protein and mRNA expression in deficient fibroblasts and is a potential therapy for fatty acid oxidation disorders. Hum Mol Genet 14:2695-2703

Ellman G (1959) Tissue sulfhydryl groups. Arch Biochem Biophys 82:70-77

Engelen M, Ofman R, Mooijer PAW, Poll-The BT, Wanders RJA, Kemp S (2008) Cholesterol-deprivation increases monounsaturated very long-chain fatty acids in skin fibroblasts from patients with X-linked adrenoleukodystrophy. Biochim Biophys Acta Mol Cell Biol 1781:105-111

Engelen M, Ofman R, Dijkgraaf MGW et al (2010) Lovastatin in XLinked Adrenoleukodystrophy. N Engl J Med 362:276-277

Islinger M, Luers GH, Li KW, Loos M, Volkl A (2007) Rat liver peroxisomes after fibrate treatment. A survey using quantitative mass spectrometry. J Biol Chem 282:23055-23069

Jakobsson A, Westerberg R, Jacobsson A (2006) Fatty acid elongases in mammals: their regulation and roles in metabolism. Progress in Lipid Research 45:237-249

Kehrer JP, Biswal SS, La E et al (2001) Inhibition of peroxisomeproliferator-activated receptor (PPAR)alpha by MK886. Biochem J 356:899-906

Kemp S, Wanders R (2010) Biochemical aspects of X-linked adrenoleukodystrophy. Brain Pathol 20:831-837

Kemp S, Mooyer PA, Bolhuis PA et al (1996) ALDP expression in fibroblasts of patients with X-linked adrenoleukodystrophy. J Inherit Metab Dis 19:667-674

Kemp S, Wei HM, Lu JF et al (1998) Gene redundancy and pharmacological gene therapy: implications for X- linked adrenoleukodystrophy. Nat Med 4:1261-1268

Kemp S, Theodoulou FL, Wanders RJ (2011) Mammalian peroxisomal $\mathrm{ABC}$ transporters: from endogenous substrates to pathology and clinical significance. Br J Pharmacol 164:1753-1766

Kishimoto Y, Moser HW, Kawamura N, Platt M, Pallante SL, Fenselau C (1980) Adrenoleukodystrophy: evidence that abnormal very long chain fatty acids of brain cholesterol esters are of exogenous origin. Biochem Biophys Res Commun 96:69-76

Miller DB, Spence JD (1998) Clinical pharmacokinetics of fibric acid derivatives (fibrates). Clin Pharmacokinet 34:155-162

Moon A, Rhead WJ (1987) Complementation analysis of fatty acid oxidation disorders. J Clin Invest 79:59-64

Moser HW, Smith KD, Watkins PA, Powers J, Moser AB (2001) Xlinked adrenoleukodystrophy. In: Scriver CR, Beaudet AL, Sly WS, Valle D (eds) The metabolic and molecular bases of inherited disease. McGraw Hill, New York, pp 3257-3301

Mosser J, Douar AM, Sarde CO et al (1993) Putative X-linked adrenoleukodystrophy gene shares unexpected homology with $\mathrm{ABC}$ transporters. Nature 361:726-730
Nagi MN, Cook L, Suneja SK et al (1989) Evidence for two separate beta-ketoacyl CoA reductase components of the hepatic microsomal fatty acid chain elongation system in the rat. Biochem Biophys Res Commun 165:1428-1434

Netik A, Forss-Petter S, Holzinger A, Molzer B, Unterrainer G, Berger J (1999) Adrenoleukodystrophy-related protein can compensate functionally for adrenoleukodystrophy protein deficiency (X-ALD): implications for therapy. Hum Mol Genet 8:907-913

Ofman R, Dijkstra IM, van Roermund CW et al (2010) The role of ELOVL1 in very long-chain fatty acid homeostasis and X-linked adrenoleukodystrophy. EMBO Mol Med 2:90-97

Pyper SR, Viswakarma N, Yu S, Reddy JK (2010) PPARalpha: energy combustion, hypolipidemia, inflammation and cancer. Nucl Recept Signal 8:e002

Rasmussen JT, Borchers T, Knudsen J (1990) Comparison of the binding affinities of acyl-CoA-binding protein and fatty-acidbinding protein for long-chain acyl-CoA esters. Biochem $\mathrm{J}$ 265:849-855

Sanchez RM, Vinals M, Alegret M et al (1993) Fibrates modify rat hepatic fatty acid chain elongation and desaturation in vitro. Biochem Pharmacol 46:1791-1796

Singh I, Khan M, Key L, Pai S (1998) Lovastatin for X-linked adrenoleukodystrophy. N Engl J Med 339:702-703

Smith PK, Krohn RI, Hermanson GT et al (1985) Measurement of protein using bicinchoninic acid. Anal Biochem 150:76-85

Tsuji S, Sano T, Ariga T, Miyatake T (1981) Increased synthesis of hexacosanoic acid (C26:0) by cultured skin fibroblasts from patients with adrenoleukodystrophy (ALD) and adrenomyeloneuropathy (AMN). J Biochem (Tokyo) 90:1233-1236

Valianpour F, Selhorst JJ, van Lint LE, van Gennip AH, Wanders RJ, Kemp S (2003) Analysis of very long-chain fatty acids using electrospray ionization mass spectrometry. Mol Genet Metab 79:189-196

van Geel BM, Assies J, Haverkort EB et al (1999) Progression of abnormalities in adrenomyeloneuropathy and neurologically asymptomatic X-linked adrenoleukodystrophy despite treatment with "Lorenzo's oil". J Neurol Neurosurg Psychiatry 67:290-299

Vazquez M, Alegret M, Lopez M et al (1995) Different effects of fibrates on the microsomal fatty acid chain elongation and the acyl composition of phospholipids in guinea-pigs. Br J Pharmacol 116:3337-3343

Vazquez M, Roglans N, Cabrero A et al (2001) Bezafibrate induces acylCoA oxidase mRNA levels and fatty acid peroxisomal betaoxidation in rat white adipose tissue. Mol Cell Biochem 216:71-78

Wanders RJ, Denis S, Ruiter JP, Schutgens RB, van Roermund CW, Jacobs BS (1995) Measurement of peroxisomal fatty acid betaoxidation in cultured human skin fibroblasts. J Inherit Metab Dis 18(Suppl 1):113-124

Watanabe T, Horie S, Yamada J et al (1989) Species differences in the effects of bezafibrate, a hypolipidemic agent, on hepatic peroxisome-associated enzymes. Biochem Pharmacol 38:367-371 